Review

\title{
Role of IDO and TDO in Cancers and Related Diseases and the Therapeutic Implications
}

\author{
Zixiang Ye, Linxiu Yue, Jiachen Shi, Mingmei Shao, Tao Wu ${ }^{\varpi}$ \\ Institute of Interdisciplinary Integrative Medicine Research, Shanghai University of Traditional Chinese Medicine, Shanghai 201203, China \\ $\triangle$ Corresponding author: Tao $\mathrm{Wu}, \mathrm{PhD}$, Assistant Professor, Institute of Interdisciplinary Integrative Medicine Research, Shanghai University of Traditional \\ Chinese Medicine, Shanghai, China. Email: tw827@shutcm.edu.cn; Telephone: +86-21-51322748; Fax: +86-21-51322748 \\ (C) Ivyspring International Publisher. This is an open access article distributed under the terms of the Creative Commons Attribution (CC BY-NC) license \\ (https://creativecommons.org/licenses/by-nc/4.0/). See http://ivyspring.com/terms for full terms and conditions.
}

Received: 2018.11.22; Accepted: 2019.04.10; Published: 2019.06.02

\begin{abstract}
Kynurenine (Kyn) pathway is a significant metabolic pathway of tryptophan (Trp). The metabolites of the Kyn pathway are closely correlated with numerous diseases. Two main enzymes, indoleamine-2,3-dioxygenase (IDO) and tryptophan-2,3-dioxygenase (TDO or TDO2), regulate the first and rate-limiting step of the Kyn pathway. These enzymes are directly or indirectly involved in various diseases, including inflammatory diseases, cancer, diabetes, and mental disorders. Presently, an increasing number of potential mechanisms have been revealed. In the present review, we depict the structure of IDO and TDO and explicate their functions in various diseases to facilitate a better understanding of them and to indicate new therapeutic plans to target them. Moreover, we summarize the inhibitors of IDO/TDO that are currently under development and their efficacy in the treatment of cancer and other diseases.
\end{abstract}

Key words: Tryptophan, Kynurenine, IDO, TDO

\section{Introduction}

Reprogrammed cell metabolism has been proven to directly cause tumorigenesis and tumor progression and other diseases [1, 2]. Therefore, targeting the abnormal metabolism would result therapeutic opportunities [2,3]. The kynurenine (Kyn) pathway is an important metabolic pathway that is closely related to many diseases through degrading tryptophan (Trp) and generating toxic Kyn metabolites. Indoleamine-2,3-dioxygenase (IDO) and tryptophan-2,3-dioxygenase (TDO, also known as TDO2) are two main enzymes involving in the first step of the Kyn pathway. IDO was extensively demonstrated in various organs throughout the body to catabolize most Trp, while TDO is mainly expressed in the liver $[4,5]$. Notably, IDO has lower substrate specificity than TDO. Recent studies have demonstrated that these two enzymes play essential biological roles as shown by their involvement in various diseases, including inflammatory diseases, cancer, diabetes and mental disorders (Figure 1).
In the present review, we aim to summarize the function of IDO and TDO involved in the Kyn pathway in different diseases and hope that it can provide some new strategies or potential targets for the prevention and therapy of cancers and related diseases.

\section{Summary of the Kyn pathway}

Trp is one of the nine essential amino acids whose L-stereoisomer participates in protein renewal and significant molecular generation, including the generation of 5-serotonin (5-HT) and metabolites of the Kyn pathway [6,7]. Trp and its catabolites of the Kyn pathway participate in forming an immunosuppressive environment to show disease tolerance and contribute to making immune privileged sites, including eyes, brain, placenta and testes $[6,7]$. Nowadays, it was recognized as a pivotal factor of innate and specific immunity via involving in infection, inflammation, and other diseases such as cancer [8]. 


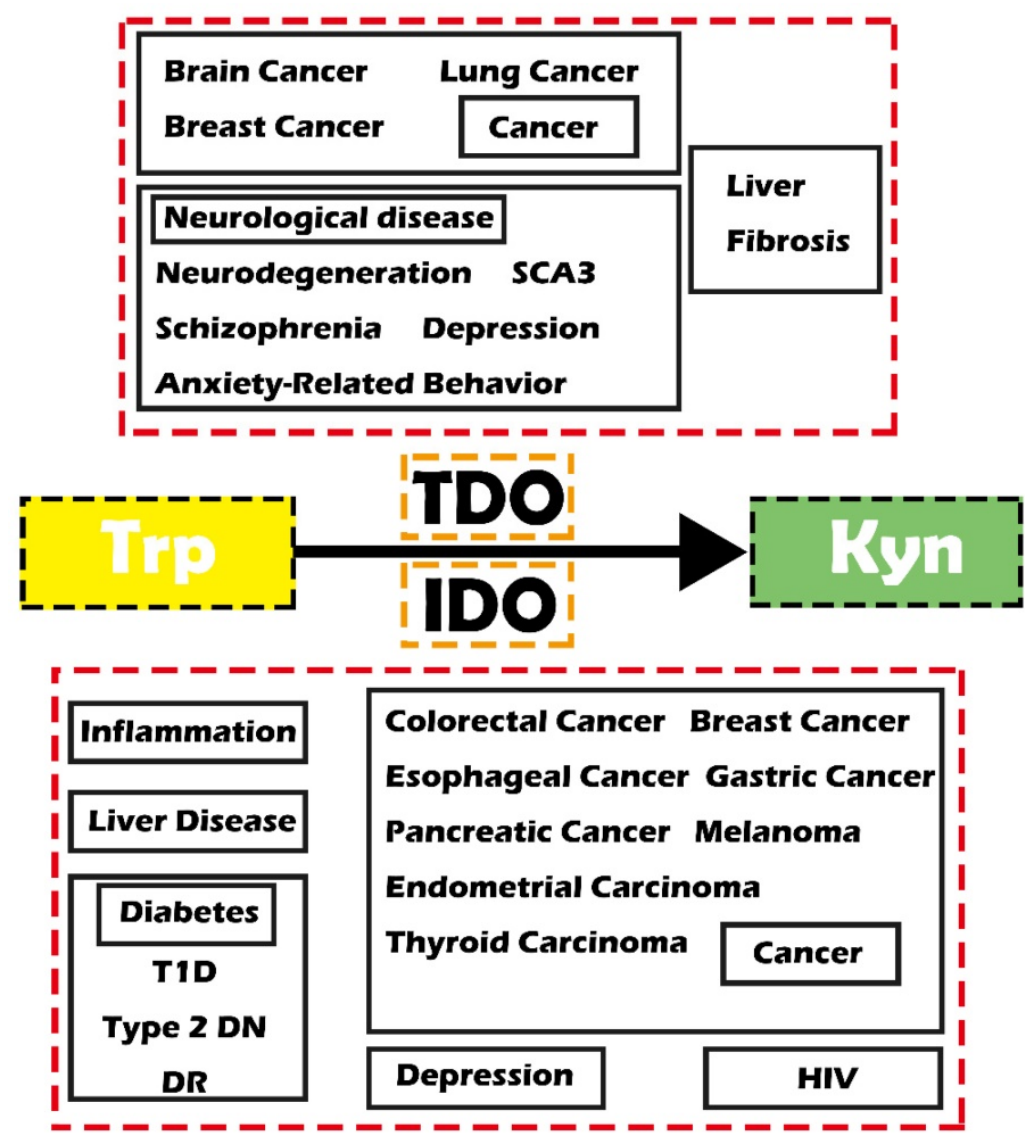

Figure 1. Schematic diagram showing IDO/TDO-related diseases. Abbreviations-Trp: tryptophan; Kyn: kynurenine; IDO: indoleamine-2,3-dioxygenase; TDO: tryptophan-2, 3-dioxygenase; SCA3: spinocerebellar ataxia type 3; TID: type 1 diabetes; Type 2 DN: type 2 diabetic nephropathy; DR: diabetic retinopathy; HIV: human immunodeficiency virus.

There are two routes of Trp decomposition: 5-HT and the Kyn pathway (Figure 2). A small subset of Trp generates 5-HT through tryptophan hydroxylase, while approximately $95 \%$ is metabolized through the Kyn pathway under the action of IDO or TDO. There are also two metabolic pathways of Kyn. One generates kynurenic acid (kynurenic acid, KYNA) directly via kynurenine aminotransferase (KAT). The other one consumes most of Kyn. Initially, Kyn generates 3-hydroxykynurenine (3-HK) under the action of kynurenine-3-monooxygenase (KMO). Next, 3-HK produces 3-hydroxyanthranilic acid (3-HAA) or xanthurenic acid (XA) by kynureninase (KYNU) or KAT separately. Finally, 3-HAA generates picolinic acid and quinolinic acid (QUIN) through multi-stage enzymatic reactions, ultimately producing nicotinamide adenine dinucleotide (NAD) as well as other active molecules involved in various physiological processes in vivo.

\section{Enzymes involved in the Kyn pathway: IDO and TDO}

Two enzymes, IDO and TDO, participate in Kyn pathway in the first and rate-limiting step [9-11]. They are found in various cells, such as tumor, stromal, vascular, and immune cells, especially antigen-presenting cells (APCs), but not expressed obviously in T cells [12]. Most Trp is catabolized by IDO, which is expressed diffusely in the human body and can be induced by interferon gamma (IFN- $\gamma$ ) [4]. For example, IFN- $\gamma$ activation of mononuclear phagocytes significantly increases IDO1 and flux through the Kyn pathway [13]. IDO has an immunomodulating function in suppressing immune activation cells and promoting immunosuppressive cells, which are related to poor survival in various cancer patients [14]. Additionally, the Kyn/Trp ratio can evaluate IDO activity [15]. TDO is located in the liver, and only works on Trp compared with the lower substrate specificity of IDO [5].

\section{Structure of IDO}

IDO is an endocellular monomeric enzyme containing heme that participates in the Kyn pathway through breaking the indole ring's 2,3-double bond in Trp when one molecular oxygen incorporates into the open molecule [16]. It produces $\mathrm{N}$-formyl-Kyn initially. Next, N-formyl-Kyn is transformed into Kyn rapidly and spontaneously. Finally, Kyn is further transformed into other active metabolites [17]. 


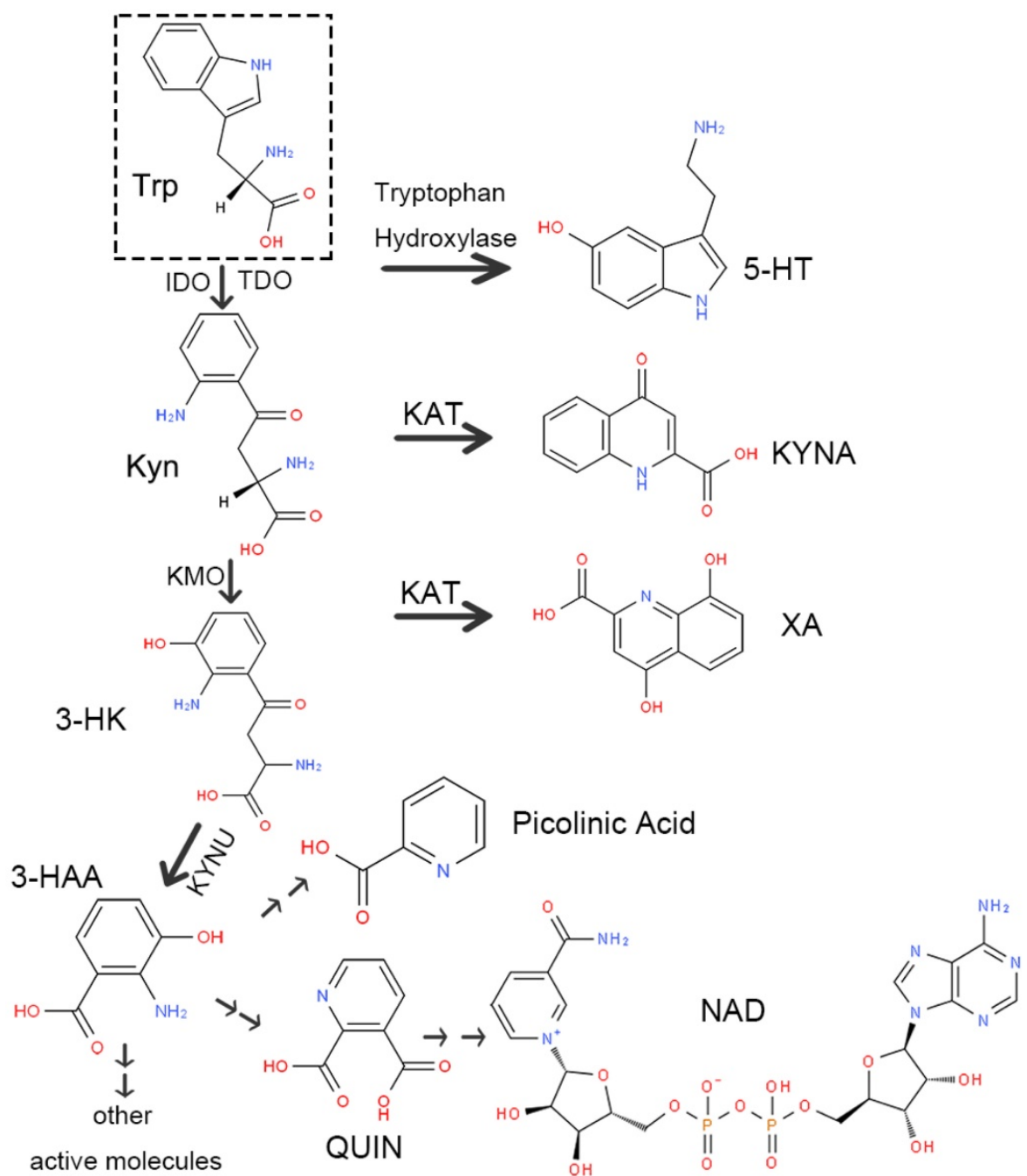

Figure 2. Schematic diagram of the kynurenine pathway. Abbreviations-Trp: tryptophan; 5-HT: 5-serotonin; Kyn: kynurenine; IDO: indoleamine-2,3-dioxygenase; TDO: tryptophan-2, 3-dioxygenase; KAT: kynurenine aminotransferase; KYNA: kynurenic acid; KMO: kynurenine-3-monooxygenase; 3-HK: 3-hydroxykynurenine; XA: xanthurenic acid; KYNU: kynureninase; 3-HAA: 3-hydroxyanthranilic acid; QUIN: quinolinic acid; NAD: nicotinamide adenine dinucleotide.

IDO was first found in rabbit intestine [18]. It has two isoforms, IDO1 and IDO2, whose genes are adjacent and have distinct biochemical features supporting their functions [18, 19]. IDO1 plays an immune-regulatory role [20]. It is a cytosolic enzyme distributed in various organs, such as the lung, spleen, liver, kidney and brain. However, IDO2 has higher specificity and much lower expression levels than IDO1 [16]. Thus, compared with IDO2, IDO1 is a research hotspot.

IDO1 is a $45-\mathrm{kDa}$ monomeric enzyme that contains heme. The $\mathrm{Fe}^{2+}$ form can activate IDO1. There are two crystal structures, 4-phenylimidazole (4PI)-bound structure and cyanide-bound structure, revealing the structural characteristics of IDO1. Furthermore, the active site of IDO1 consists of two oleophilic areas: one is a large container containing heme as the catalytic site of Trp, the other is the binding site entrance [19].
IDO1 activity is modulated by two phosphorylatable tyrosine residues, Y115 and Y253. These residues can change the conformation of IDO1 and discourage its activity via phosphorylation. Additionally, they are binding sites to prolong or shorten the IDO1 half-life and maintain its functions [16]. Through a range of kinetic, structural and cellular assays, Nelp et al. [21] showed two highly selective inhibitors of IDO1 taking effect by competing with heme for binding to apo-IDO1. This result implies that IDO1 is bound to its heme cofactor dynamically and is likely a critical step in the regulation of this enzyme.

IDO1 can be expressed in many cells, including astrocytes, macrophages and dendritic cells (DCs) [22]. Tumor cells can also express widely but variably IDO1, such as the tumor microenvironment's innate immune cells and tumor-draining lymph nodes [12]. IDO1 can be induced during infection, where it displays antimicrobial activity and immune 
regulation [23]. Moreover, some inflammatory cytokines, such as interleukin (IL) 6 and tumor necrosis factor- $\alpha$ (TNF- $\alpha$ ), can highly induce IDO1 [22].

\section{Structure of TDO}

TDO is a tetrameric hemoprotein encoded by the TDO2 gene [24]. It also catalyzes the transformation of $\mathrm{L}$-Trp to $\mathrm{N}$-formyl-Kyn via adding $\mathrm{O}_{2}$ to the 2,3-bond of Trp's indole ring [25, 26].

TDO was first found in mammalian liver in 1936 and was characterized in rat liver in 1955 [27]. It has been characterized in many prokaryotes and eukaryotes, including rabbits, mice, some insects such as Drosophila melanogaster, and bacteria such as Xanthomonas campestris and yeast [27]. In the human body, high TDO was demonstrated in the liver, and TDO supports up to $95 \%$ of hepatic Trp metabolism [28]. Moreover, TDO can be detected in the kidney [29], skin [27] and other tissues, including the placenta, pregnant uterus, epididymis, testis and brain after being stimulated [30].

TDO can be activated by cortisol [31] and it was also revealed that TDO can be activated by Trp-induced reactive oxygen species [24]. TDO comprises four non-covalent bonding subunits that have the same mass and contain two heme parts in every molecule [32]. Its heme group is present as an inactive $\mathrm{Fe}^{3+}$ form that needs to be changed to $\mathrm{Fe}^{2+}$ form to be active[24]. Furthermore, TDO is mainly found as homodimers that can transform to active tetramers of $\sim 190 \mathrm{kDa}$ in eukaryotes and $\sim 120 \mathrm{kDa}$ in prokaryotes [27, 33]. Lewis-Ballester et al. [34] found a binding site for L-Trp in hTDO that did not influence enzyme catalysis but can block the degradation of hTDO, revealing a new L-Trp-mediated regulation mechanism for cellular hTDO degradation. Additionally, Michels et al. [33] found that three amino acid residues, TDO-2 ( $\triangle \mathrm{PLD}), \mathrm{TDO}-2$ (del), and TDO-2 (del B), were vital for the enzymatic activity of TDO.

\section{Function of IDO or TDO in different diseases}

The function of IDO and TDO can be direct or indirect regarding the pathogenesis of cancer and other diseases (Table 1).

\section{Function of IDO in different diseases}

Increased IDO activity is related to many diseases, such as inflammatory diseases, cancer, liver diseases, diabetes, depression, HIV and rejection of organ transplants [16].

IDO1 plays an important role in immunologic processes and this role was first found in pregnancy
$[35,36]$. Affected by maternal $\mathrm{T}$ cells, pregnant mice given IDO1 inhibitors showed allogeneic fetal rejection, reflecting that IDO1 prevented maternal $\mathrm{T}$ cells from injuring fetal allografts. Moreover, IDO1 serves as an immunosuppressant that facilitates the formation of immunosuppressive environments through promoting the tolerogenicity of APCs and death of immune cells [22]. IDO1 promotes the tolerogenicity of APCs via acting as a direct intracellular signaling molecule that is independent of its enzymatic activity [12]. Furthermore, IDO1 is not restricted to IDO1-expressing cells to show its immunologic functions. In fact, it can indirectly affect surrounding cells through the Kyn pathway [18].

At the protein level, besides activating the aryl hydrocarbon receptor (AhR), IDO1 can activate general control non-derepressible 2 (GCN2) to suppress T cells. GCN2 activation can directly change DCs to tolerogenic APCs and stimulate tumor immune-inhibitory cytokines such as IL-10 and transforming growth factor beta (TGF- $\beta$ ) to restrain effector $\mathrm{T}$ cells and upgrade regulatory cells (Tregs) to stimulate tolerance, and $\mathrm{AhR}$ can promote Treg differentiation and make DCs and macrophages tend to an immunosuppressive phenotype $[36,37]$.

\section{IDO and Inflammation}

IDO function is based on inflammatory programming, hence influencing tumor neovascularization, myeloid-derived suppressor cells (MDSC) generation, and metastasis of adaptive immune tolerance [12].

Many chronic inflammatory syndromes show increased IDO1 activity including cancer, infections, allergic diseases, and transplant rejection because of the response to interferons [36]. IDO1 consumes Trp and produces Kyn metabolites to help attenuate inflammation and maintain tissue homeostasis [38]. Ogiso et al. [39] demonstrated that IDO1 blocked the functions of inflammatory cells and stimulated immunologic tolerance. They found that IDO1 shortage stimulated early liver regeneration after partial hepatectomy, reflecting that the production of inflammatory cytokines and liver proliferation could be blocked by IDO1. Additionally, IDO1-expressing DCs, active Tregs and Trp catabolites cooperate to suppress infection in inflammation [22]. Interestingly, IDO1 can downregulate IFN- $\gamma$ and upregulate IL-6 at the early stage of tumorigenesis to transform the inflammatory environments to immunosuppressive environments [12]. IDO2 is responsible for inflammatory autoimmunity. Merlo et al. [40] revealed that the activation of autoreactive $\mathrm{T}$ and $\mathrm{B}$ cells, pathogenic autoantibody production and rheumatoid arthritis development were induced by 
IDO2. Remarkably, IDO2 expressed in B cells can regulate the function of $\mathrm{B}$ cells via interacting with $\mathrm{T}$ cells. The role of IDO2 in inflammatory autoimmunity implies that IDO2 is, to some extent, the opposite of IDO1.

Table 1. The role of IDO and TDO in diseases.

\begin{tabular}{|c|c|c|c|c|}
\hline IDO/TDO & Disease & Impact & Mechanism & Reference \\
\hline IDO2 & $\begin{array}{l}\text { Rheumatoid } \\
\text { arthritis }\end{array}$ & Aggravate & $\begin{array}{l}\uparrow \text { Inflammatory } \\
\text { autoimmunity }\end{array}$ & $\begin{array}{l}\text { Merlo et al., } \\
2017\end{array}$ \\
\hline \multirow[t]{8}{*}{ IDO1 } & Fn-associated CRC & Attenuate & $\uparrow$ Kyn pathway & $\begin{array}{l}\text { Xue et al., } \\
2018\end{array}$ \\
\hline & Breast Cancer & Aggravate & $\begin{array}{l}\downarrow \text { APCs } \uparrow \text { Kyn } \\
\text { pathway } \\
\uparrow \text { MDSCs }\end{array}$ & $\begin{array}{l}\text { Wei et al., } \\
2018\end{array}$ \\
\hline & Melanoma & Aggravate & $\begin{array}{l}\downarrow \text { APCs } \uparrow \text { Kyn } \\
\text { pathway } \\
\uparrow \text { MDSCs }\end{array}$ & $\begin{array}{l}\text { Rubel et al., } \\
2018\end{array}$ \\
\hline & Gastric Cancer & Aggravate & $\begin{array}{l}\downarrow \text { APCs } \uparrow \text { Kyn } \\
\text { pathway } \\
\uparrow \text { MDSCs }\end{array}$ & $\begin{array}{l}\text { Nishi et al., } \\
2018\end{array}$ \\
\hline & Pancreatic Cancer & Aggravate & $\begin{array}{l}\downarrow \text { APCs } \uparrow \text { Kyn } \\
\text { pathway } \\
\uparrow \text { MDSCs }\end{array}$ & $\begin{array}{l}\text { Zhang et al., } \\
2017\end{array}$ \\
\hline & $\begin{array}{l}\text { Endometrial } \\
\text { Carcinomas }\end{array}$ & Aggravate & $\begin{array}{l}\downarrow \text { APCs } \uparrow \text { Kyn } \\
\text { pathway } \\
\uparrow \text { MDSCs }\end{array}$ & $\begin{array}{l}\text { Mills et al., } \\
2018\end{array}$ \\
\hline & $\mathrm{EC}$ & Aggravate & $\begin{array}{l}\downarrow \text { APCs } \uparrow \text { Kyn } \\
\text { pathway } \\
\uparrow \text { MDSCs }\end{array}$ & $\begin{array}{l}\text { Ari et al., } \\
2018 ; \\
\text { Cui et al., } \\
2018\end{array}$ \\
\hline & $\begin{array}{l}\text { Thyroid } \\
\text { Carcinomas }\end{array}$ & Aggravate & $\begin{array}{l}\downarrow \text { APCs } \uparrow \text { Kyn } \\
\text { pathway } \\
\uparrow \text { MDSCs }\end{array}$ & $\begin{array}{l}\text { Moretti et } \\
\text { al., } 2017\end{array}$ \\
\hline \multirow[t]{3}{*}{ TDO } & Brain Tumors & Aggravate & $\uparrow$ Kyn pathway & $\begin{array}{l}\text { Ochs et al., } \\
2016\end{array}$ \\
\hline & Lung Cancer & Aggravate & $\uparrow$ Kyn pathway & $\begin{array}{l}\text { Ya-Ling } \\
\text { Hsu et al., } \\
2016\end{array}$ \\
\hline & Breast Cancer & Aggravate & $\uparrow$ Kyn pathway & $\begin{array}{l}\text { D'Amato et } \\
\text { al., } 2015\end{array}$ \\
\hline \multirow[t]{3}{*}{ IDO1 } & TID & Attenuate & $\uparrow$ Trp catabolism & $\begin{array}{l}\text { Orabona et } \\
\text { al., } 2018\end{array}$ \\
\hline & Type 2 DN & Aggravate & & $\begin{array}{l}\text { Zhang et al., } \\
2017\end{array}$ \\
\hline & DR & Aggravate & $\begin{array}{l}\uparrow \text { Retinal } \\
\text { endothelial } \\
\text { apoptosis } \\
\uparrow \text { Capillary } \\
\text { degeneration }\end{array}$ & $\begin{array}{l}\text { Nahomi et } \\
\text { al., } 2018\end{array}$ \\
\hline IDO1 & Depression & Aggravate & & Thomas et \\
\hline \multirow[t]{2}{*}{ TDO } & Depression & Aggravate & $\uparrow$ Kyn pathway & $\begin{array}{l}\text { al., 2017; } \\
\text { Merete et } \\
\text { al., 2008; } \\
\text { Campayo et } \\
\text { al., } 2010\end{array}$ \\
\hline & & & $\uparrow$ Kyn pathway & $\begin{array}{l}\text { Michels et } \\
\text { al., 2016; } \\
\text { Gibney et } \\
\text { al., } 2014\end{array}$ \\
\hline IDO1 & HIV & Aggravate & $\begin{array}{l}\downarrow \text { CD4+ immune } \\
\text { cells }\end{array}$ & $\begin{array}{l}\text { Chen et al., } \\
2018\end{array}$ \\
\hline \multirow[t]{4}{*}{ TDO } & Neurodegeneration & Aggravate & & $\begin{array}{l}\text { Wu et al., } \\
\text { 2013; } \\
\text { Breda et al., } \\
2016\end{array}$ \\
\hline & SCA3 & Aggravate & $\uparrow$ QUIN & $\begin{array}{l}\text { Rajamani et } \\
\text { al., } 2017\end{array}$ \\
\hline & Schizophrenia & Aggravate & & $\begin{array}{l}\text { Miller et al., } \\
2004\end{array}$ \\
\hline & $\begin{array}{l}\text { Anxiety-Related } \\
\text { Behavior }\end{array}$ & Aggravate & & $\begin{array}{l}\text { Kanai et al., } \\
2009\end{array}$ \\
\hline IDO1 & Liver Fibrosis & Aggravate & $\uparrow$ Th17 & $\begin{array}{l}\text { Zhong et } \\
\text { al., } 2017\end{array}$ \\
\hline TDO & & Attenuate & $\begin{array}{l}\downarrow \text { Th17 } \downarrow \text { CD8+ } \\
\text { T cells }\end{array}$ & $\begin{array}{l}\text { Zhong et } \\
\text { al., } 2017\end{array}$ \\
\hline
\end{tabular}

\begin{tabular}{|c|c|c|c|c|}
\hline \multirow[t]{2}{*}{ IDO1 } & \multirow[t]{2}{*}{$\mathrm{HCV}$} & $\begin{array}{l}\text { Attenuate acute } \\
\text { infection phase }\end{array}$ & $\begin{array}{l}\uparrow \text { Antiviral } \\
\text { defense }\end{array}$ & \multirow[t]{2}{*}{$\begin{array}{l}\text { Yang et al., } \\
2018\end{array}$} \\
\hline & & $\begin{array}{l}\text { Aggravate chronic } \\
\text { infection stage }\end{array}$ & $\begin{array}{l}\downarrow \text { Anti-HCV T } \\
\text { cells }\end{array}$ & \\
\hline
\end{tabular}

Abbreviation. IDO: indoleamine-2,3-dioxygenase; TDO:

tryptophan-2,3-dioxygenase; Fn: fusobacterium nucleatum; CRC: colorectal Cancer; Kyn: kynurenine; APCs: antigen-presenting cells; MDSCs: myeloid-derived suppressor cells; EC: esophageal cancer; T1D: type 1 diabetes; Trp: tryptophan; Type 2 DN: type 2 diabetic nephropathy; DR: diabetic retinopathy; HIV: human immunodeficiency virus; SCA3: spinocerebellar ataxia type 3; QUIN: quinolinic acid; Th17: $\mathrm{T}$ helper 17 cells; $\mathrm{HCV}$ : hepatitis $\mathrm{C}$ virus

\section{IDO and Cancer}

In terms of cancer, many studies have investigated IDO1, but few have focused on IDO2. A poor prognosis of various cancers has been shown to be related to high IDO1 expression [19]. IDO1 facilitates cancer via forming immune tolerance. It can be inhibited by the tumor suppressor Bin1, which is attenuated in human tumors [12]. Moreover, various cancer cells and APCs show that high IDO1 levels and IDO1 can be induced by IL-6, IFN- $\gamma$, TGF- $\beta$, cytotoxic T-lymphocyte-associated protein 4 (CTLA-4) and programmed cell death protein 1 (PD-1) [4, 41]. IDO1 suppresses the immune system to aggravate cancer cell growth, migration and invasion in three ways.

Initially, IDO1 promotes the formation of immunosuppressive APCs via acting as a direct intracellular signaling molecule that is independent of its enzymatic activity [20]. It makes APCs tolerogenic to promote tumorigenesis and help build immune checkpoints in cancer [36]. For example, in tumor draining lymph nodes, IDO1 expressed in APCs can enhance the peripheral tolerance to tumor-associated antigens (TAAs), keeping the TAA-expressing malignant cells active [17].

Secondly, the induction of IDO1 in APCs indirectly regulates adjacent immune cells through the Kyn pathway. It blocks natural killer (NK) cells and CD8+T effector cells but induces CD4+ regulatory cells (iTreg) and MDSCs [12]. All of them do not express IDO1 obviously. Activation of the Kyn pathway induces AhR and activates GCN2, inhibits mammalian target of rapamycin (mTOR) via accelerating Kyn production and Trp consumption, which inhibits CD8+ T cells and induces Tregs [12, 41]. Additionally, Trp consumption can also inhibit NK cells and T-cell proliferation in the G1 phase, while some toxic Kyn metabolites can cause T-cell apoptosis and selectively suppress T-helper 17 cells (Th17) [22, 42]. It is worth mentioning that IDO1-regulated iTregs and B-cell inflammation states in cancer need the support of IDO2 [12].

Finally, MDSCs can suppress T cells and NK cells, as well as facilitate the migration of cancer. IDO1 induces MDSCs via influencing inflammatory condition, such as activating IL-6. MDSCs can migrate to tumor tissues to create an immunosuppressive 
tumor microenvironment. Additionally, IDO1 promotes angiogenesis to facilitate cancer growth via downregulating IFN- $\gamma$ and upregulating IL-6 [12].

Altogether, IDO1 might enhance the proliferation, invasion, and metastatic abilities of cancer cells to represent a high degree of malignancy via three ways: (1) Tolerogenic APCs: IDO1 promotes the tolerogenicity of APCs to promote tumorigenesis and help build immune checkpoints in cancer as well as influence adjacent cells [34]; (2) Activating the Kyn pathway: Overexpression of IDO1 in APCs induces the Kyn pathway to facilitate Kyn release and Trp consumption. The formation of Kyn can activate AhR, and the consumption of Trp leads to the induction of GCN2 and inhibition of mTOR, which suppresses CD8+ T cells and NK cells and induces Tregs [9, 39, 41, 42]. Moreover, toxic Kyn pathway metabolites such as 3-HAA, QUIN, and picolinic acid, can inhibit T-cell activity and induce the dissolution and apoptosis of thymocytes and $\mathrm{T}$ cells [43, 44]. (3) MDSCs induction: IDO facilitates MDSCs suppressive function by changing inflammation condition, such as activating IL-6, to suppress CD8+T cells and NK cells as well as induce cancer migration [13].

\section{IDOI and Colorectal Cancer (CRC)}

Fusobacterium nucleatum (Fn) is a tumor-associated obligate anaerobic bacterium that promotes metastasis in CRC. Xue et al. [43] reported the link between IDO1 and Fn in THP-1-derived macrophages (dTHP1). They found that IDO1-induced low Trp and high Kyn environment inhibited the intracellular multiplication of $\mathrm{Fn}$ in dTHP1 cells. The function of peripheral blood lymphocytes that permitted the escape of Fn-infected macrophages from cell death was further impaired by IDO1 expression. These findings indicate that the inhibition of IDO1 may worsen infection in Fn-associated tumor immunotherapy.

\section{IDOI and Breast Cancer}

Wei et al. [44] measured IDO1 mRNA expression in breast cancer cell lines and the concentration of Trp and Kyn in the supernatants of MCF-7. They found that IDO1 facilitated the angiogenesis in breast cancer. Patterson [45] found that estrogen receptor alpha (ER) negatively regulated IDO1 expression. After analyzing 14 breast cancer data sets, they found that, in ER-positive tumors, IDO1 expression was lower than that in ER-negative cells. Finally, they speculated that ER may suppress IDO1 via facilitating the methylation of the IDO1 promoter.

\section{IDOI and Melanoma}

In primary human melanoma cells, Rubel et al. [46] reported that IDO1 expression was related to
Breslow thickness, tumor-infiltrating lymphocytes and the intensity of the peritumoral inflammatory infiltrate.

\section{IDOI and Gastric Cancer}

Nishi et al. [20] found that IDO1 expression was correlated with TGF- $\beta$ expression, and, in stage III gastric cancer patients who underwent curative resection, TGF- $\beta$ expression was correlated with Foxp3 expression.

\section{IDOI and Pancreatic Cancer}

In pancreatic cancer, Zhang et al. [47] confirmed that high IDO1 expression was related to a poor prognosis, and patients with low IDO1 expression showed better survival.

\section{IDOI and Endometrial Carcinomas}

IDO1 can be expressed in endometrial carcinoma. Mills et al. [48] assessed IDO1 expression in 60 endometrial carcinomas and the relationship with programmed death-1(PD-L1). They finally concluded that IDO1 expression was widespread in endometrial carcinomas, and diffuse staining was common in tumors that lacked mismatch repair.

\section{IDOI and Esophageal Cancer (EC)}

In EC, patients who showed high levels of IDO1 and PD-L1 showed a worse survival and had more T cells [49]. In esophageal squamous cell carcinoma (ESCC), Cui et al. [50] found the high expression of IDO1 that was mainly expressed by ESCC cells and stromal cells. They demonstrated that IDO1 expression was greatly influenced by reactive tumor-associated fibroblasts and capillary in the ESCC, which could explain the ability of ESCC cells in suppressing antitumor immunity and sustaining their growth and progression.

\section{IDOI and Thyroid Carcinoma}

In thyroid carcinoma, Moretti et al. [51] reported high IDO1 expression. They found that BRAF point mutations and RET gene rearrangement (RET/PTC) oncogenes induced IDO1, leading to overexpressed and phosphorylated signal transducer and activator of transcription 1 (STAT1), which could predict the occurrence of thyroid carcinoma. Conversely, STAT1 activation would occur during cancer progression with the absence of RET/PTC rearrangements. Altogether, IDO1 overexpression driven by STAT1 ultimately protects tumors from the immune system and maintains their growth.

\section{IDOI and Liver Diseases}

Zhong et al. found that IDO1 is a marker of liver diseases such as liver fibrosis and cirrhosis. They 
reported that IDO1-dificient mice did not develop $\mathrm{CCl}_{4}$-induced fibrosis via reducing Th17 cells and compensating TDO [52]. They also noted that the degree of cirrhosis was positively correlated with IDO1. Furthermore, the IDO1 level was positively related to serum alanine transaminase (ALT), aspartate transaminase (AST), $\gamma$-glutamyl transferase (GGT), total bilirubin (TB), indirect bilirubin (IB), direct bilirubin (DB) and total bile acid (TBA) levels. However, it showed a negative correlation with serum albumin (ALB) levels, the albumin/globulin (A/G) ratio, cholinesterase (CHE) levels and prothrombin time activity (PTA) levels.

Yang et al. [22] revealed that plasma IDO1 is related to TGF- $\beta$ and the degree of chronic hepatitis $C$ virus (HCV) infection. In acute infection stage, IDO1 was upregulated and facilitated the antiviral defense progress. However, in chronic infection stage, IDO1 was upregulated by IFN- $\gamma$ in HCV-infected liver cells to suppress the anti-HCV T cells.

\section{IDOI and Diabetes}

\section{IDOI and Type 1 Diabetes (TID)}

IDO1 displays an immunosuppressive function, thus it can be used to treat certain autoimmune diseases, such as T1D, which is recognized by a remarkable defect in Trp catabolism. Orabona et al. [53] found that, in NOD mice, IDO1 deficiency impaired the immune tolerance to autoantigens, suggesting that upregulating IDO1 may resist T1D.

IDOI and Type 2 Diabetic Nephropathy (Type 2 DN)

Inflammation and endoplasmic reticulum stress can regulate IDO1. IDO1 participates in the development of type 2 DN. Zhang et al. [35] found that, in type 2 DN patients, IDO1 activity was much higher than that in individuals with latent glomerulonephritis but much lower than that in those with maintenance hemodialysis. The results revealed that IDO1 activity had a negative correlation with estimated glomerular filtration rate and had a positive correlation with the severity of chronic kidney disease.

\section{IDOI and Diabetic Retinopathy (DR)}

Nahomi et al. [54] reported that IDO1 played an important role in retinal endothelial apoptosis and capillary degeneration in the diabetic retina. They summarized four molecular mechanisms of IDO1 in endothelial cell apoptosis: (1) hyperglycemia enhanced IFN-c-mediated IDO1 expression; (2) increased IFN-c induced IDO1 in the retinal capillary endothelium; (3) oxidative stress regulated IDO1-mediated endothelial cell apoptosis; and (4)
IDO1 deficiency in diabetic reduced the formation of acellular capillary.

\section{IDOI and Depression}

It was reported that IDO1 can also drive depression. It was also reported that, in IFN-a associated depression, IDO1 activity was elevated by IFN-a, facilitating the development of depression[22]. Thomas et al. [55] also found that neurotoxic Kyn pathway metabolites can cause major depressive disorders. Some evidence has suggested that vitamin B6 deficiency could lead to depression [56, 57], likely due to increased XA which is a metabolite of the Kyn pathway. Vitamin B6 also serves as the coenzyme of KYNU.

\section{IDOI and Human Immunodeficiency Virus (HIV)}

Chen et al. [58] found that IDO1 activity has a positive relationship with HIV DNA in the blood of patients who received antiretroviral therapy, indicating that IDO1 participates in the persistence of HIV. It is possible that the immunosuppressive function of IDO1 can protect HIV from elimination and help them to duplicate via suppressing CD4+ immune cells.

\section{The function of TDO in different diseases}

TDO is mainly present in the liver and metabolizes $95 \%$ of hepatic Trp. It is a key serotonergic determinant [59] and plays a key role in the emotional and psychiatric status [60]. Due to the similar enzymatic function with IDO, it can also suppress inflammation and maintain immune homeostasis [61] by Trp starvation [62]. Schmidt et al. [63] found that the TDO in HeLa cells could inhibit certain bacteria, parasites and viruses. In animal models, TDO was also reported to suppress disease phenotypes and promote survive [33]. Additionally, Elbers et al. [64] reported that, under hypoxic conditions, the ability of TDO to inhibit pathogens and T-cell proliferation could be attenuated.

\section{TDO and Cancer}

It was reported that cancer tissues express IDO1 or TDO, or both [65]. Many studies have reported that TDO could be expressed in various cancers, such as brain cancers, lung cancers and breast cancers [25].

Due to the similar roles of TDO and IDO in regulating the Kyn pathway, TDO was definitely considered a potential therapeutic target to cancer. TDO has been proven to have immunomodulatory functions in promoting tumor immune resistance and proliferation [66]. Some data have suggested that cancer cells overexpressing TDO and activating AhR can evade immune surveillance [62]. 


\section{TDO and Brain Tumors}

The TDO-AhR pathway also participates in brain tumors and is correlated with the development of cancers and a poor survival [25]. Ochs et al. [67] reported that, in malignant gliomas, prostaglandins activated prostaglandin $\mathrm{E}$ receptor-4 (EP4) to upregulate TDO and strengthen its activity. EP4 and prostaglandin E2- producing cyclooxygenase-2 influence the expression of TDO at the transcript and protein levels.

\section{TDO and Lung Cancer}

Ya-Ling et al. [68] reported that lung fibroblasts that were induced by lung cancer could produce Kyn to inhibit DC differentiation and induce cancer growth and migration. Moreover, they found that lung cancer-derived galectin-1 contributed to upregulating TDO in cancer-associated fibroblasts through the AKT-dependent pathway.

\section{TDO and Breast Cancer}

D'Amato et al. [69] suggested that TDO was the important factor in aggressive breast cancer subtype, and high TDO in primary breast tumors was related to shorter overall survival. They also found that it was possible that the effects of TDO could be mediated partly via other intermediate molecule production or $\mathrm{NAD}+$ production.

\section{TDO and Neurological Diseases}

There is an increasing number of patients with neurodegenerative diseases such as Alzheimer's (AD), Parkinson's (PD) and Huntington's (HD) that correlate with Trp degradation in the Kyn pathway. TDO can be expressed in neurons and the brain vasculature[25], and its deficiency is beneficial for resisting mental disorders [70]. Breda et al [71] used fruit fly models to show that inhibiting TDO at the gene level and influencing neuroactive Kyn pathway metabolites can alleviate these diseases. They even found that some compounds that inhibited TDO could reverse disease phenotypes, highlighting the importance of the Kyn pathway in treating neurodegenerative disease.

\section{TDO and Neurodegeneration}

The Kyn pathway is related to neurodegeneration diseases such as $\mathrm{AD}, \mathrm{PD}$ and $\mathrm{HD}$ [25]. TDO is thought to participate in these disease pathways via regulating proteotoxic events [33]. Wu et al. [72] observed significantly high TDO immunoreactivity in the AD patients' hippocampus. In fly models of $\mathrm{AD}$ and PD, Breda et al. [71] found that inhibiting TDO could improve motor performance and help survival, and they also used the
HD fruit fly model to find that increased KYNA relative to $3-\mathrm{HK}$ could alleviate $\mathrm{HD}$, indicating that shifting the flux in the Kyn pathway to KYNA synthesis rather than $3-\mathrm{HK}$ synthesis could reduce neurodegeneration.

\section{TDO and Spinocerebellar Ataxia Type 3 (SCA3)}

TDO is a key enzyme for neuronal toxicity in SCA3. Rajamani et al.[73] found that upregulation of TDO occurred in the SCA3 mouse cerebellum. QUIN, a metabolite of the Kyn pathway, is a neurotoxic compound that can activate N-methyl-D-aspartate receptors. It allows $\mathrm{Ca}^{2+}$ ions to flood into neuronal cells, thus increasing calpain activity and toxic compounds as well as suppressing calpain inhibitors to result in neuronal excitotoxicity.

\section{TDO and Schizophrenia}

Miller et al. [74] showed increased TDO-immunopositive astroglial cells in the white matter of schizophrenia patients by using immunohistochemistry.

\section{TDO and Anxiety-Related Behavior}

In TDO-defective mice, Kanai et al. [75] found that, in plasma, there was more Trp, 5-hydroxyindoleacetic acid (5-HIAA) and Kyn; however, in the hippocampus and midbrain, there was more Trp, serotonin and 5-HIAA. These mice showed good performance on the elevated plus maze and increased adult neurogenesis, suggesting that TDO had a negative correlation with anxiety-related behavior.

\section{TDO and Depression}

5-HT has a negative correlation with the degree of depression. It also mediates Trp metabolism and the Kyn pathway. Thus, TDO has a positive correlation with the degree of depression[33]. Gibney et al. [76] used adult male Sprague-Dawley rats that had restraint stress per $2 \mathrm{~h}$ to research the relationship of TDO and depression via increasing glucocorticoid corticosterone to induce TDO. These mice showed increased immobility that had a positive correlation with the degree of depression. Accompanied with increased immobility, there was increased circulating glucocorticoid corticosterone and hepatic and cerebral cortex TDO, suggesting that inhibiting stress-related TDO could suppress depression.

\section{TDO and Liver Fibrosis}

Zhong et al. [77] found that compensatory increased TDO due to IDO1 deficiency could attenuate $\mathrm{CCl}_{4}$-induced liver fibrosis. They hypothesized that increased TDO in the liver induced by IDO1 deficiency could suppress Th17 cells 
proliferation. They also supposed that increased TDO consumed more Trp to decrease CD8+ $\mathrm{T}$ cells that could attack injured liver cells.

\section{The inhibitors of IDO or TDO in treatment}

With the function of IDO and TDO in promoting cancer, there is an increasing number of their inhibitors being developed to treat cancer. Due to the effect of PD-1 and CTLA-4 on inducing IDO, IDO inhibitors can combine with PD-1 and CTLA-4 inhibitors to strengthen efficacy. Although most of the inhibitors have been focused on treating cancer, their regulation of the Kyn pathway may also be considered to treat other Trp- or Kyn-related metabolic diseases.

\section{Ipilimumab}

The CTLA-4 blocking antibody, ipilimumab, can inhibit IDO because its expression can be induced by the CTLA-4 pathway. Treating patients with ipilimumab with higher IDO expression in host stromal cells in the tumor has good efficacy [41].

\section{Indoximod (D-1MT/NLG-8189)}

Indoximod is an inhibitor of IDO and TDO. It may act partly as the Trp analog to restore the attenuation of mTOR resulting from the induction of IDO and TDO in Trp consumption. Additionally, its low toxicity and better efficacy combined with taxotere, dendritic cell vaccine sipuleucel- $\mathrm{T}$ and anti-PD1 make indoximod attractive in clinical settings [12].

\section{1-L-MT}

Liu et al.[78] reported that 1-L-MT, an inhibitor of IDO, can be used to prevent and treat cancer because of its ability to suppress cancer cell proliferation via inhibiting mitosis. It decreases CDC20 transcription to cause G2/M cycle arrest and facilitates mitochondrial injuries as well as cancer cell apoptosis.

\section{Epacadostat (INCB024360)}

Epacadostat can selectively inhibit IDO1. In addition to inhibiting IDO1, it can also suppress IDO2 and TDO slightly. Epacadostat administration can decrease Kyn levels and inhibit cancer outgrowth. Moreover, the efficacy can be strengthened when combined with PD-L1 or CTLA-4 inhibitors.

\section{BMS-986205}

BMS-986205 is an IDO1 inhibitor that can be taken orally with little effect on IDO2 and TDO. Current clinical studies have shown that the efficacy can be increased with a dose increase. Even at a low dose, it can show good ability in inhibiting IDO1 and serum Kyn levels [37]. It was reported to have better efficacy and pharmacokinetics than epacadostat [12].

\section{LW106}

LW106 is an IDO inhibitor that shows better antineoplastic effect than epacadostat [79]. It can reduce Tregs and non-hematopoietic stromal cells, as well as cancer stem cells (CSCs) in the tumor microenvironment, to inhibit cancer growth. Moreover, it can be used with immune checkpoint inhibitors or chemotherapeutic drugs.

\section{Ethylnaphthoquinone Derivatives}

Cui et al. [80] isolated and identified ethylnaphthoquinone derivatives in the mangrove endophytic fungus N. austral SYSU-SKS024. It was the first time to discover a novel inhibiting IDO carbon skeleton that showed a good effect.

\section{4-Bromophenylhydrazinyl benzenesulfonylphenylureas}

Lin et al. [81] developed 4-bromophenylhydrazinyl benzenesulfonylphenylurea that was good at inhibiting IDO. Its oral administration could inhibit $25 \%$ cancer growth and reduce the tumor weight by $30 \%$. Additionally, it could also decrease Kyn and Trp levels and may restore immunity.

\section{Navoximod (NLG919)}

Navoximod can inhibit IDO via binding to heme iron at the active site. It has abilities to decrease Kyn and restore T-cell functions. Furthermore, the therapeutic effect can be strengthened when combined with PD-L1 inhibitors [12].

\section{PF-06840003}

PF-06840003 is a nonheme-binding IDO1 inhibitor. It was reported to have excellent pharmacokinetics and central nervous penetration and can be administered as a single dose daily with good effects on brain cancer. Additionally, it can be combined with PD-1 or PD-L1 inhibitors to enhance the curative effect [12].

\section{4-amino-1,2,3-triazole inhibitors}

Alexandre et al. [82] found a series of new 4-amino-1,2,3-triazole inhibitors that bound to apo-IDO1. They combined with IDO1 to form an extremely tight and long-lived complex that may be the core of their excellent efficacy.

\section{TDO inhibitors}

Compared with IDO inhibitors, the development of TDO inhibitors is relatively backward. Most of them are in the stage of exploring 
and modifying structures, such as naphthotriazolediones [83], 3-(indol-3-yl)pyridine derivatives [25], aminoisoxazoles [61] and 3-(2-(Pyridyl)ethenyl)indoles [84]. Due to the higher expression of TDO than IDO in some diseases, TDO inhibitors warrant more attention.

\section{Prospects and Problems}

The strategy to selectively inhibit IDO1 or TDO, or to dually inhibit IDO1 and TDO, needs to be considered within the realm of personalized medicine to avoid unnecessary side effects or inadequate target engagement. A recent review provides the newest progress in targeting the metabolites of Kyn to be used for cancer immunotherapy, and they proposed the upcoming challenges and opportunities to target the Kyn pathway for anticancer immunotherapies [62]. Compared with IDO1, IDO2 is more recognized in inflammatory autoimmunity. Little is known about IDO2 in the immune system, but some evidence has suggested that it can support IDO1 to mediate Tregs [12]. It implies that, except being responsible for inflammatory autoimmunity, it may play a role in helping IDO1 participate in the immunologic process, which needs further investigation. However, it is still a long way to specifically illustrate the detailed mechanism of those enzymes such as IDO1, IDO2, and TDO, which play important roles in different diseases such as cancers. There is an increasing number of IDO and TDO inhibitors being developed, but most of them are IDO inhibitors and treat cancer. Due to the effect of the Kyn pathway on other related diseases, using IDO and TDO inhibitors may also be efficacious. Furthermore, the development of TDO inhibitors may have great prospects.

\section{Acknowledgements}

This work was supported by the National Natural Science Foundation of China (81873076), Shanghai Rising-Star Project (15QA1403500) and Shanghai Talents development fund Project (2017090).

\section{Competing Interests}

The authors have declared that no competing interest exists.

\section{References}

1. Vander Heiden MG, DeBerardinis RJ. Understanding the Intersections between Metabolism and Cancer Biology. Cell. 2017; 168: 657-69.

2. Halbrook CJ, Lyssiotis CA. Employing Metabolism to Improve the Diagnosis and Treatment of Pancreatic Cancer. Cancer Cell. 2017; 31: 5-19.

3. Martinez-Outschoorn UE, Peiris-Pages M, Pestell RG, et al. Cancer metabolism: a therapeutic perspective. Nat Rev Clin Oncol. 2017; 14: 11-31.

4. Werner ER, Bitterlich G, Fuchs D, et al. Human macrophages degrade tryptophan upon induction by interferon-gamma. Life Sci. 1987; 41: 273-80.
5. Capece L, Arrar M, Roitberg AE, et al. Substrate stereo-specificity in tryptophan dioxygenase and indoleamine 2,3-dioxygenase. Proteins. 2010; 78: 2961-72.

6. Moffett JR, Namboodiri MA. Tryptophan and the immune response. Immunol Cell Biol. 2003; 81: 247-65.

7. Thackray SJ, Mowat CG, Chapman SK. Exploring the mechanism of tryptophan 2,3-dioxygenase. Biochem Soc Trans. 2008; 36: 1120-3.

8. O'Mahony SM, Clarke G, Borre YE, et al. Serotonin, tryptophan metabolism and the brain-gut-microbiome axis. Behav Brain Res. 2015; 277: 32-48.

9. Mehler AH, Knox WE. The conversion of tryptophan to kynurenine in liver. II. The enzymatic hydrolysis of formylkynurenine. J Biol Chem. 1950; 187: 431-8.

10. Lob S, Konigsrainer A, Zieker D, et al. IDO1 and IDO2 are expressed in human tumors: levo- but not dextro-1-methyl tryptophan inhibits tryptophan catabolism. Cancer Immunol Immunother. 2009; 58: 153-7.

11. Metz R, Duhadaway JB, Kamasani U, et al. Novel tryptophan catabolic enzyme IDO2 is the preferred biochemical target of the antitumor indoleamine 2,3-dioxygenase inhibitory compound D-1-methyl-tryptophan. Cancer Res. 2007; 67: 7082-7.

12. Prendergast GC, Malachowski WJ, Mondal A, et al. Indoleamine 2,3-Dioxygenase and Its Therapeutic Inhibition in Cancer. Int Rev Cell Mol Biol. 2018; 336: 175-203.

13. Grant RS. Indoleamine 2,3-Dioxygenase Activity Increases NAD+ Production in IFN-gamma-Stimulated Human Primary Mononuclear Cells. Int J Tryptophan Res. 2018; 11: 1-8.

14. Ferns DM, Kema IP, Buist MR, et al. Indoleamine-2,3-dioxygenase (IDO) metabolic activity is detrimental for cervical cancer patient survival. Oncoimmunology. 2015; 4: e981457.

15. Creelan BC, Antonia S, Bepler G, et al. Indoleamine 2,3-dioxygenase activity and clinical outcome following induction chemotherapy and concurrent chemoradiation in Stage III non-small cell lung cancer. Oncoimmunology. 2013; 2: e23428.

16. Hornyak L, Dobos N, Koncz G, et al. The Role of Indoleamine-2,3-Dioxygenase in Cancer Development, Diagnostics, and Therapy. Front Immunol. 2018; 9: 151.

17. Zhang G, Xing J, Wang $Y$, et al. Discovery of Novel Inhibitors of Indoleamine 2,3-Dioxygenase 1 Through Structure-Based Virtual Screening. Front Pharmacol. 2018; 9: 277.

18. Chang RQ, Li DJ, Li MQ. The role of indoleamine-2,3-dioxygenase in normal and pathological pregnancies. Am J Reprod Immunol. 2018; 79: e12786.

19. Weng $\mathrm{T}$, Qiu $\mathrm{X}$, Wang $\mathrm{J}$, et al. Recent discovery of indoleamine-2,3-dioxygenase 1 inhibitors targeting cancer immunotherapy. Eur J Med Chem. 2018; 143: 656-69.

20. Nishi M, Yoshikawa K, Higashijima J, et al. The Impact of Indoleamine 2,3-dioxygenase (IDO) Expression on Stage III Gastric Cancer. Anticancer Res. 2018; 38: 3387-92.

21. Nelp MT, Kates PA, Hunt JT, et al. Immune-modulating enzyme indoleamine 2,3-dioxygenase is effectively inhibited by targeting its apo-form. Proc Natl Acad Sci U S A. 2018; 115: 3249-54.

22. Yang R, Gao N, Chang Q, et al. The role of IDO, IL-10 and TGF-beta in the HCV-associated chronic hepatitis, liver cirrhosis, and hepatocellular carcinoma. J Med Virol. 2018.

23. Zardoya-Laguardia P, Blaschitz A, Hirschmugl B, et al. Endothelial indoleamine 2,3-dioxygenase-1 regulates the placental vascular tone and is deficient in intrauterine growth restriction and pre-eclampsia. Sci Rep. 2018; 8: 5488 .

24. Li JS, Han Q, Fang JM, et al. Biochemical mechanisms leading to tryptophan 2,3-dioxygenase activation. ARCH INSECT BIOCHEM. 2006; 64: 74-87.

25. Abdel-Magid AF. Targeting the Inhibition of Tryptophan 2,3-Dioxygenase (TDO-2) for Cancer Treatment. ACS Med Chem Lett. 2017; 8: 11-3.

26. Fukumura E, Sugimoto H, Misumi $Y$, et al. Cooperative binding of L-trp to human tryptophan 2,3-dioxygenase: resonance Raman spectroscopic analysis. J Biochem. 2009; 145: 505-15.

27. Rafice SA, Chauhan N, Efimov I, et al. Oxidation of L-tryptophan in biology: a comparison between tryptophan 2,3-dioxygenase and indoleamine 2,3-dioxygenase. Biochem Soc Trans. 2009; 37: 408-12.

28. Jernigan FE, Sun LJ. In silico discovery and therapeutic potential of IDO1 and TDO2 inhibitors. FUTURE MED CHEM. 2017; 9: 1309-11.

29. Dang YH, DALE WE, BROWN OR. Comparative effects of oxygen on indoleamine 2,3-dioxygenase and tryptophan 2,3-dioxygenase of the kynurenine pathway. FREE RADICAL BIO MED. 2000; 28: 615-24.

30. Pantouris G, Mowat CG. Antitumour agents as inhibitors of tryptophan 2,3-dioxygenase. Biochem Biophys Res Commun. 2014; 443: 28-31.

31. Navrotskaya V, Oxenkrug G, Vorobyova L, et al. Attenuation of high sucrose diet-induced insulin resistance in tryptophan 2,3-dioxygenase 
deficient Drosophila melanogaster vermilion mutants. Integr Obes Diabetes. 2015; 1: 93-5.

32. Dick R, Murray BP, Reid MJ, et al. Structure--function relationships of rat hepatic tryptophan 2,3-dioxygenase: identification of the putative heme-ligating histidine residues. Arch Biochem Biophys. 2001; 392: 71-8.

33. Michels H, Seinstra RI, Uitdehaag JC, et al. Identification of an evolutionary conserved structural loop that is required for the enzymatic and biological function of tryptophan 2,3-dioxygenase. Sci Rep. 2016; 6: 39199.

34. Lewis-Ballester A, Forouhar F, Kim SM, et al. Molecular basis for catalysis and substrate-mediated cellular stabilization of human tryptophan 2,3-dioxygenase. Sci Rep. 2016; 6: 35169.

35. Zhang $Y$, Ruan $Y$, Zhang $P$, et al. Increased indoleamine 2,3-dioxygenase activity in type 2 diabetic nephropathy. J Diabetes Complications. 2017; 31: 223-7.

36. Mellor $\mathrm{AL}$, Lemos $\mathrm{H}$, Huang L. Indoleamine 2,3-Dioxygenase and Tolerance: Where Are We Now? Front Immunol. 2017; 8: 1360.

37. Davar D, Bahary N. Modulating Tumor Immunology by Inhibiting Indoleamine 2,3-Dioxygenase (IDO): Recent Developments and First Clinical Experiences. Target Oncol. 2018; 13: 125-40.

38. Salazar F, Awuah D, Negm OH, et al. The role of indoleamine 2,3-dioxygenase-aryl hydrocarbon receptor pathway in the TLR4-induced tolerogenic phenotype in human DCs. Sci Rep. 2017; 7: 43337.

39. Ogiso $\mathrm{H}$, Ito $\mathrm{H}$, Kanbe $\mathrm{A}$, et al. The Inhibition of Indoleamine 2,3-Dioxygenase Accelerates Early Liver Regeneration in Mice After Partial Hepatectomy. Dig Dis Sci. 2017; 62: 2386-96.

40. Merlo LMF, Grabler S, DuHadaway JB, et al. Therapeutic antibody targeting of indoleamine-2,3-dioxygenase (IDO2) inhibits autoimmune arthritis. Clin Immunol. 2017; 179: 8-16.

41. Munn DH, Mellor AL. Indoleamine 2,3 dioxygenase and metabolic control of immune responses. Trends Immunol. 2013; 34: 137-43.

42. Song $\mathrm{X}$, Zhang $\mathrm{Y}$, Zhang $\mathrm{L}$, et al. Hypoxia enhances indoleamine 2,3-dioxygenase production in dendritic cells. Oncotarget. 2018; 9: $11572-80$.

43. Xue $\mathrm{Y}$, Xiao $\mathrm{H}$, Guo S, et al. Indoleamine 2,3-dioxygenase expression regulates the survival and proliferation of Fusobacterium nucleatum in THP-1-derived macrophages. Cell Death Dis. 2018; 9: 355.

44. Wei L, Zhu S, Li M, et al. High Indoleamine 2,3-Dioxygenase Is Correlated With Microvessel Density and Worse Prognosis in Breast Cancer. Front Immunol. 2018; 9: 724.

45. Dewi DL, Mohapatra SR, Cabañes SB, et al. Suppression of indoleamine-2,3-dioxygenase 1 expression by promoter hypermethylation in ER-positive breast cancer. Oncoimmunology. 2017; 6: e1274477.

46. Rubel F, Kern JS, Technau-Hafsi K, et al. Indoleamine 2,3-Dioxygenase Expression in Primary Cutaneous Melanoma Correlates with Breslow Thickness and Is of Significant Prognostic Value for Progression-Free Survival. J Invest Dermatol. 2018; 138: 679-87.

47. Zhang T, Tan XL, Xu Y, et al. Expression and Prognostic Value of Indoleamine 2,3-dioxygenase in Pancreatic Cancer. Chin Med J (Engl). 2017; 130: 710-6.

48. Mills A, Zadeh S, Sloan E, et al. Indoleamine 2,3-dioxygenase in endometrial cancer: a targetable mechanism of immune resistance in mismatch repair-deficient and intact endometrial carcinomas. Mod Pathol. 2018

49. Rosenberg AJ, Wainwright DA, Rademaker A, et al. Indoleamine 2,3-dioxygenase 1 and overall survival of patients diagnosed with esophageal cancer. Oncotarget. 2018; 9: 23482-93.

50. Cui G, Li C, Xu G, et al. Tumor-Associated Fibroblasts and Microvessels Contribute to the Expression of Immunosuppressive Factor Indoleamine 2, 3-Dioxygenase in Human Esophageal Cancers. Pathol Oncol Res. 2018; 24: 269-75.

51. Moretti S, Menicali E, Nucci N, et al. Signal Transducer and Activator of Transcription 1 Plays a Pivotal Role in RET/PTC3 Oncogene-induced Expression of Indoleamine 2,3-Dioxygenase 1. J Biol Chem. 2017; 292: 1785-97.

52. Zhong $\mathrm{W}, \mathrm{Gao} \mathrm{L}, \mathrm{Zhou} \mathrm{Z}$, et al. Indoleamine 2,3-dioxygenase 1 deficiency attenuates CCl4-induced fibrosis through Th17 cells down-regulation and tryptophan 2,3-dioxygenase compensation. Oncotarget. 2017; 8: 40486-500.

53. Orabona C, Mondanelli G, Pallotta MT, et al. Deficiency of immunoregulatory indoleamine 2,3-dioxygenase 1in juvenile diabetes. JCI Insight. 2018; 3: 1-17.

54. Nahomi RB, Sampathkumar S, Myers AM, et al. The Absence of Indoleamine 2,3-Dioxygenase Inhibits Retinal Capillary Degeneration in Diabetic Mice. Invest Ophthalmol Vis Sci. 2018; 59: 2042-53.
55. Thomas I, Khanam R, Vohora D. Activation of indoleamine 2, 3dioxygenase pathway by olanzapine augments antidepressant effects of venlafaxine in mice. Psychiatry Res. 2017; 258: 444-8.

56. Merete C, Falcon LM, Tucker KL. Vitamin B6 is associated with depressive symptomatology in Massachusetts elders. Journal of the American College of Nutrition. 2008; 27: 421-7.

57. Campayo A, de Jonge P, Roy JF, et al. Depressive disorder and incident diabetes mellitus: the effect of characteristics of depression. The American journal of psychiatry. 2010; 167: 580-8.

58. Chen J, Xun J, Yang J, et al. Plasma indoleamine 2,3-dioxygenase activity is associated with the size of HIV reservoir in patients receiving antiretroviral therapy. Clin Infect Dis. 2018

59. Liao M, Pabarcus MK, Wang Y, et al. Impaired dexamethasone-mediated induction of tryptophan 2,3-dioxygenase in heme-deficient rat hepatocytes: translational control by a hepatic eIF2alpha kinase, the heme-regulated inhibitor. J Pharmacol Exp Ther. 2007; 323: 979-89.

60. Kanai M, Nakamura T, Funakoshi H. Identification and characterization of novel variants of the tryptophan 2,3-dioxygenase gene: differential regulation in the mouse nervous system during development. Neurosci Res. 2009; 64: 111-7.

61. Pei Z, Mendonca R, Gazzard L, et al. Aminoisoxazoles as Potent Inhibitors of Tryptophan 2,3-Dioxygenase 2 (TDO2). ACS Med Chem Lett. 2018; 9: 417-21.

62. Cheong JE, Sun L. Targeting the IDO1/TDO2-KYN-AhR Pathway for Cancer Immunotherapy - Challenges and Opportunities. Trends Pharmacol Sci. 2018; 39: 307-25.

63. Schmidt SK, Muller A, Heseler $\mathrm{K}$, et al. Antimicrobial and immunoregulatory properties of human tryptophan 2,3-dioxygenase. Eur J Immunol. 2009; 39: 2755-64.

64. Elbers F, Woite C, Antoni V, et al. Negative Impact of Hypoxia on Tryptophan 2,3-Dioxygenase Function. Mediators Inflamm. 2016; 2016: 1638916.

65. Liu X, Shin N, Koblish HK, et al. Selective inhibition of IDO1 effectively regulates mediators of antitumor immunity. Blood. 2010; 115: 3520-30.

66. Pantouris G, Loudon-Griffiths J, Mowat CG. Insights into the mechanism of inhibition of tryptophan 2,3-dioxygenase by isatin derivatives. J Enzyme Inhib Med Chem. 2016; 31: 70-8.

67. Ochs K, Ott M, Rauschenbach KJ, et al. Tryptophan-2,3-dioxygenase is regulated by prostaglandin E2 in malignant glioma via a positive signaling loop involving prostaglandin E receptor-4. J Neurochem. 2016; 136: $1142-54$

68. Hsu YL, Hung JY, Chiang SY, et al. Lung cancer-derived galectin-1 contributes to cancer associated fibroblast-mediated cancer progression and immune suppression through TDO2kynurenine axis. Oncotarget. 2016; 7: 27584-98.

69. D'Amato NC, Rogers TJ, Gordon MA, et al. A TDO2-AhR signaling axis facilitates anoikis resistance and metastasis in triple-negative breast cancer. Cancer Res. 2015; 75: 4651-64.

70. Lanz TV, Williams SK, Stojic A, et al. Tryptophan-2,3-Dioxygenase (TDO) deficiency is associated with subclinical neuroprotection in a mouse model of multiple sclerosis. Sci Rep. 2017; 7: 41271

71. Breda C, Sathyasaikumar KV, Sograte Idrissi S, et al. Tryptophan-2,3-dioxygenase (TDO) inhibition ameliorates neurodegeneration by modulation of kynurenine pathway metabolites. Proc Natl Acad Sci U S A. 2016; 113: 5435-40.

72. $\mathrm{Wu} \mathrm{W}$, Nicolazzo JA, Wen L, et al. Expression of tryptophan 2,3-dioxygenase and production of kynurenine pathway metabolites in triple transgenic mice and human Alzheimer's disease brain. PLoS One. 2013; 8: e59749.

73. Rajamani $\mathrm{K}$, Liu JW, Wu CH, et al. n-Butylidenephthalide exhibits protection against neurotoxicity through regulation of tryptophan 2, 3 dioxygenase in spinocerebellar ataxia type 3. Neuropharmacology. 2017; 117: $434-46$

74. Miller CL, Llenos IC, Dulay JR, et al. Expression of the kynurenine pathway enzyme tryptophan 2,3-dioxygenase is increased in the frontal cortex of individuals with schizophrenia. Neurobiol Dis. 2004; 15: 618-29.

75. Kanai M, Funakoshi H, Takahashi H, et al. Tryptophan 2,3-dioxygenase is a key modulator of physiological neurogenesis and anxiety-related behavior in mice. Mol Brain. 2009; 2: 8 .

76. Gibney SM, Fagan EM, Waldron AM, et al. Inhibition of stress-induced hepatic tryptophan 2,3-dioxygenase exhibits antidepressant activity in an animal model of depressive behaviour. Int J Neuropsychopharmacol. 2014; 17: 917-28.

77. Zhong WC, Gao L, Zhou ZT, et al. Indoleamine 2,3-dioxygenase 1 deficiency attenuates CCl4-induced fibrosis through Th17 cells down-regulation and tryptophan 2,3-dioxygenase compensation. Oncotarget. 2017; 8: 40486-500. 
78. Liu X, Zhou W, Zhang X, et al. 1-L-MT, an IDO inhibitor, prevented colitis-associated cancer by inducing CDC20 inhibition-mediated mitotic death of colon cancer cells. Int J Cancer. 2018; 143: 1516-29.

79. Fu R, Zhang YW, Li HM, et al. LW106, a novel indoleamine 2,3-dioxygenase 1 inhibitor, suppresses tumour progression by limiting stroma-immune crosstalk and cancer stem cell enrichment in tumour micro-environment. Br J Pharmacol. 2018; 175: 3034-49.

80. Cui H, Zhang H, Liu Y, et al. Ethylnaphthoquinone derivatives as inhibitors of indoleamine-2, 3-dioxygenase from the mangrove endophytic fungus Neofusicoccum austral SYSU-SKS024. Fitoterapia. 2018; 125: 281-5.

81. Lin SY, Yeh TK, Song JS, et al. 4-Bromophenylhydrazinyl benzenesulfonylphenylureas as indoleamine 2,3-dioxygenase inhibitors with in vivo target inhibition and anti-tumor efficacy. Bioorg Chem. 2018; 77: 600-7.

82. Alexandre JAC, Swan MK, Latchem MJ, et al. New 4-Amino-1,2,3-Triazole Inhibitors of Indoleamine 2,3-Dioxygenase Form a Long-Lived Complex with the Enzyme and Display Exquisite Cellular Potency. Chembiochem. 2018; 19: 552-61.

83. Wu JS, Lin SY, Liao FY, et al. Identification of Substituted Naphthotriazolediones as Novel Tryptophan 2,3-Dioxygenase (TDO) Inhibitors through Structure-Based Virtual Screening. J Med Chem. 2015; 58: 7807-19.

84. Dolusic E, Larrieu P, Moineaux L, et al. Tryptophan 2,3-dioxygenase (TDO) inhibitors. 3-(2-(pyridyl)ethenyl)indoles as potential anticancer immunomodulators. J Med Chem. 2011; 54: 5320-34. 